\title{
Synovial sarcoma presenting as a neck mass, report of two cases and review of literature
}

\author{
Che-Wei Wang ${ }^{1}$ and Chin-Fang Chang $2,3,4 *$ \\ ${ }^{1}$ Department of Otorhinolaryngology, Head and Neck Surgery, Changhua Christian Hospital, Changhua, Taiwan \\ ${ }^{2}$ Department of Otorhinolaryngology, Head and Neck Surgery, Jen-Ai Hospital, Taichung, Taiwan \\ ${ }^{3}$ Central Taiwan University of Science and Technology, Taichung, Taiwan \\ ${ }^{4}$ School of medicine, Chung Shan Medical University, Taichung, Taiwan
}

\section{Summary}

Synovial sarcoma is a rare malignant tumor of mesenchymal origin. We would like to present two cases of synovial sarcomas of the parotid region and submandibular space. A detailed review of the literature on synovial sarcoma is also included.

\section{Introduction}

Synovial sarcoma is a rare malignant tumor of mesenchymal origin. It accounts for approximately $8 \%(5.6 \%-10 \%)$ of all the soft tissue sarcoma, showing a specific chromosomal translocation $t(X ; 18)$ (p11; q11) [1]. It arises most commonly in the deep soft tissues of lower extremities such as the knee and ankle. Male adolescents and young adults are especially affected. Only $3 \%$ to $10 \%$ of all synovial sarcomas occur in the head and neck region, the pharyngeal and cervical areas are most commonly affected while the larynx is the least common site.

Primary synovial sarcomas of the salivary glands are exceptionally uncommon, with few cases reported in the literature. Diagnosis depends on histological examination, and the gold standard for the diagnosis of SS is the detection of translocation $\mathrm{t}(\mathrm{X}: 18)$ by polymerase chain reaction (PCR) or fluorescence in situ hybridization (FISH) [1]. Surgical wide excision is the main idea of therapy although the tumor is sensitive to chemotherapy and radiotherapy. The prognosis depends on the size and grade of the tumor [2]. We would like to present two cases of synovial sarcomas of the parotid region and submandibular space. A detailed review of the literature on synovial sarcoma is also included.

\section{Case report}

\section{Case 1}

A 35-year-old lady presented with a 2-year history of a slow-growth painless mass over left submandibular space. At the same time, no facial palsy, headache, epistaxis and left deafness was mentioned. CT scan showed a homogenous mass up to $1.6 \times 2.9 \mathrm{~cm}$ was noted in left level II area without adjacent fat stranding (Figure 1). Ultrasonographic examination revealed a $3.37 \times 3.03 \times 1.68 \mathrm{~cm}$ irregular hypoechoic mass over left submandibular area. An ultrasoundguided fine-needle aspiration biopsy was performed and malignancy was highly suspected from pathological report. Thus, wide excision of the tumor was performed. Besides, postoperative radiotherapy and chemotherapy were arranged to strength local control and prevent the occurrence of distant metastases. After operation the specimen was examined, grossly the tumor and the submandibular gland are separated. Section of the tumor showed lobulated mass with firm texture and gray color. Histopathologic examination revealed that a spindle cell tumor composed of densely cellular sheets and fascicles with occasional nuclear palisading (Figure 2). Immunohistochemical study for CK, EMA, S-100, CD34 and CD68 is performed and is non-revealing. Special stain for reticulin and PTAH is also non-revealing. The result of RT-PCR assay shows presence of SYT-SSX1 gene fusion, confirming the diagnosis of synovial sarcoma. Postoperative MRI at $12^{\text {th }}$ month showed no evidence of recurrence (Figure 3 ).

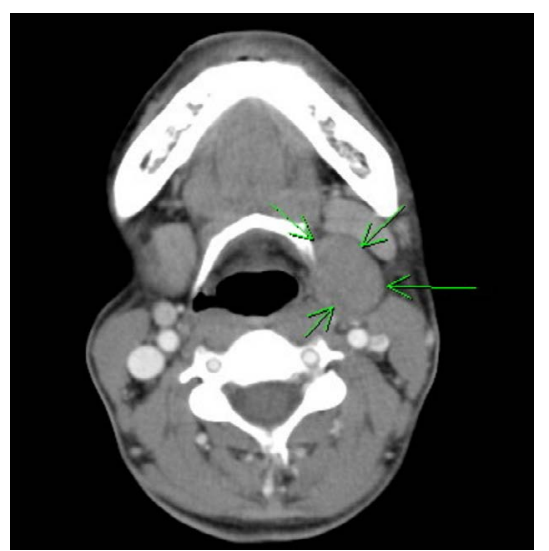

Figure 1. CT scan before operation, a homogenous tumor was noted in left level II area.

Correspondence to: Chin-Fang Chang, M.D., M.S. Department of Otorhinolaryngology, Head and Neck Surgery, Jen-Ai Hospital, \#483, Dongrong Rd., Dali Dist., Taichung City 412, Taiwan, Tel: +886-4-2481-9900; E-mail: benglung@hotmail.com

Key words: synovial sarcoma; SYT-SSX1 gene

Received: July 06, 2016; Accepted: August 16, 2016; Published: August 19, 2016 


\section{Case 2}

A 20-year-old man presented with a mild tender mass over right infra-auricular region for three months. On examination, no facial palsy, headache, epistaxis and left deafness was noted. CT scan showed $2.75 \mathrm{~cm}$ mild hypodense nodular lesion in right level II showed welldefined margin and solid heterogenous content, no calcififcation or fatty content (Figure 4). Incision biopy was done and the pathology report suspected perivascular or myofibroblastic tumor. Thus, surgery for wide excision of the tumor was done. Because the frozen section in operation showed suspection of malignant sarcoma, neck dissection of suprahyoid level was performed at the same time. After operation, the

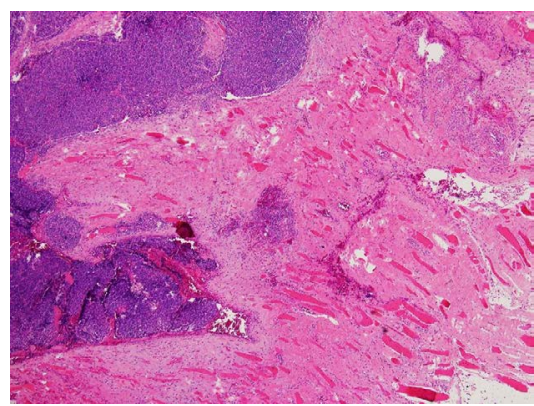

Figure 2. The tumor within parotid gland composed of highly cellular whirling fascicles of spindle-shaped cells with indistinct cytoplasms (haematoxylineeosin stain,original magnification $\mathrm{x} 100$ ).

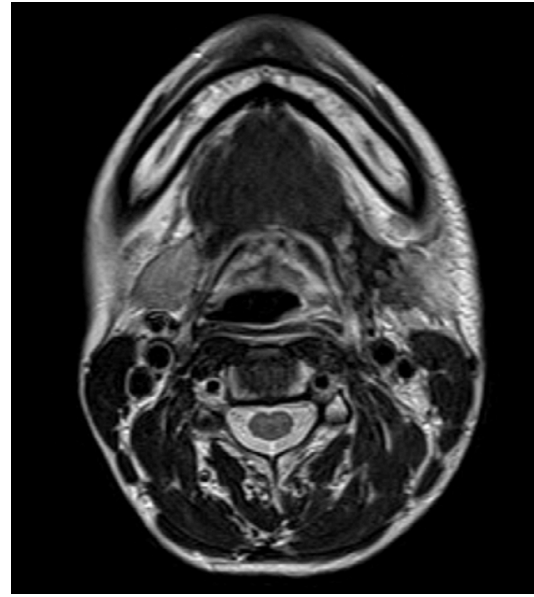

Figure 3. MRI at 12 months post-operation, no obvious local recurrence was noted.

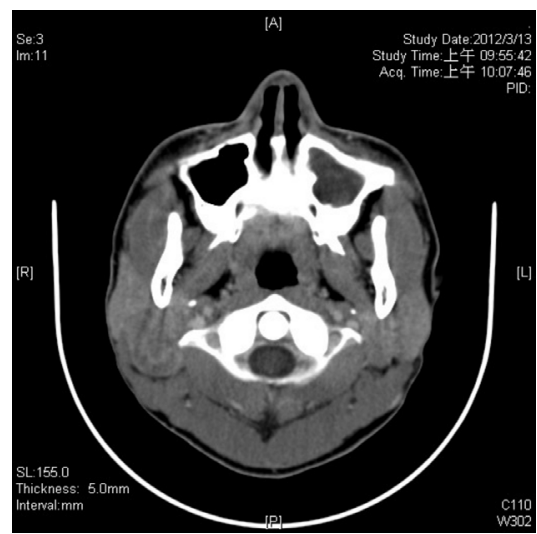

Figure 4. $\mathrm{CT}$ scan before the second patient received surgery; a tumor was noted right submandibular area. molecular study shows presence of SYT-SSX1 gene fusion and synovial sarcoma without neck metastasis was diagnosed. Postoperative radiotherapy of dose $6400 \mathrm{cGy}$ was arranged to strength local control. After 13 months of follow up, no evidence of local recurrence or distal metastasis was noted.

\section{Discussion}

Synovial sarcoma is the fourth most common variety of sarcoma after malignant fibrous histiocytoma, liposarcoma and rhabdomyosarcoma. This mesenchymal spindle cell malignancy was first proposed by Knox [3], because the tumor showed a resemblance to synovial tissue. There are two cell subtypes of synovial sarcoma: monophasic and biphasic. In $99 \%$ patients with synovial sarcoma, they present a special gene translocation between chromosome 18 and X result in SYT-SSX1 or SYT-SSX2 gene fusion. This translocation thus makes it easy to confirm the diagnosis of synovial sarcoma. The American Joint Committee on Cancer has classified all synovial sarcomas as high grade malignancies, irrespective of their phenotypes.

Synovial sarcoma arises most commonly in the deep soft tissues of lower extremities, especially in the region of the lower thigh. Only 5\% occur within the joints. The peak incidence is in adolescents and young adults between 16 and 49 years of age (median 34 years), and the tumor affects predominant in males [4]. It has been reported that patients with synovial sarcoma are generally a decade younger in the head and neck group. The most common site in head and neck is the hypopharynx, with the larynx being the least common site. The typical clinical feature of SS in the head and neck tends to present as an asymptomatic mass, but in some literatures patients presented with the onset of painful neck mass. Its clinical features lead to differential diagnoses of neck masses in adolescents, such as inflammatory lymphadenopathy, branchial cysts, dermoid cysts, and thyroglossal duct cysts, and also benign mesenchymal tumors, such as schwannoma, neurofibroma, and lipoma.

Primary sarcomas of the major salivary glands are rare, accounting for approximately $3 \%$ of malignant salivary gland tumors [4]. Primary synovial sarcoma of the parotid gland is exceptionally uncommon, with few cases reported in the literature.Primary synovial sarcoma of the parotid gland is a rare occurrence that may commonly cause differential diagnostic difficulties. In the parotid gland, it is most likely to be misdiagnosed as amyoepithelial, primary mesenchymal, or metastatic neoplasm. A diagnosis of Synovial sarcoma was established based on the histology and, in particular, the positivity for AE1/AE3, CK8/18, EMA, and CD99 and the strong reaction to CD56 and TLE1. Recently, reverse-transcription PCR or FISH for t(X;18) (p11; q11) was necessary to prove a diagnosis of Synovial sarcoma.

Many authors agree that tumor size is the most important factor of prognosis. Patients with tumors greater than $5 \mathrm{~cm}$ in diameter have a poorer prognosis than those with tumors less than $5 \mathrm{~cm}$ in diameter. Harb et al. say that survival rates were related to tumor location, size, and extension. The most common site of distant metastasis is the lung (97\%), in some cases it may occur years after initial therapy, with an average duration of 30.8 months from initial treatment [5-7]. Kartha and Bumpous [8] stressed a more aggressive behavior of the synovial sarcoma of the head and neck than of the same neoplasm in the extremities. Thus, it is no doubt that multidisciplinary approach in the treatment ofthis disease.

Surgery followed by radiotherapy with or without chemotherapy has been advocated by most studies. However, there is no evidence 
support the sole treatment modality of chemotherapy or radiotherapy. A prophylactic neck dissection is not known to have a benefit for the survival rate since synovial sarcomas rarely show lymphatic spread [810]. No standardized treatment protocol for now is available for this type of tumor. The behavior of synovial sarcoma is variable, with a $50 \%$ recurrent rate within 2 years and late recurrences have also been noted even after 30 years. Several reports have suggested an aggressive course for head and neck synovial sarcoma. A review of 36 cases in the head and neck revealed local recurrences in $20.8 \%$, and metastasis to the lungs, liver, pleura, bone, retroperitoneum, and mediastinum in 29.2\% [8]. Follow-up should be lifelong due to the tendency for late recurrences and metastasis. In our second case, the clinical appearance and the preliminary histopathological results misled us at first to the diagnosis of a benign parotid tumor [8]. Because the frozen section in operation showed highly suspected unconcerned malignancy, we performed suprahyoid neck dissection. After confirming the presence of a synovial sarcoma the application of adjuvant therapy was discussed.

Synovial sarcomas are rare in the head and neck region. They can mimic benign lesions clinically as well as histologically. In this report we presented that synovial sarcomas should be considered as a differential diagnosis for uncertain neck mass. Sometimes genetic testing might be required to confirm the diagnosis. As more cases are reported it will hopefully lead to a better understanding of synovial sarcomas in the head and neck region. We will continue to follow up on these patients.

\section{References}

1. Rigante M, Visocchi M, Petrone G, Mulè A, Bussu F (2011) Synovial sarcoma of the parotid gland: a case report and review of the literature. Acta Otorhinolaryngol Ital 31: 43-46. [Crossref]

2. Spillane AJ, A'Hern R, Judson IR, Fisher C, Thomas JM (2000) Synovial sarcoma: a clinicopathologic, staging, and prognostic assessment. J Clin Oncol 18: 3794-3803. [Crossref]

3. Luksic I, Virag M, Manojlovic S, Obradovic B, Macan D, et al. (2011) Primary synovial sarcoma of the parotid gland in 15-year-old boy. J Craniomaxillofac Surg 39: 445-448. [Crossref]

4. Kusuma S, Skarupa DJ, Ely KA, Cmelak AJ, Burkey BB (2010) Synovial sarcoma of the head and neck: a review of its diagnosis and management and a report of a rare case of orbital involvement. Ear Nose Throat J 89: 280-283. [Crossref]

5. Al-Daraji W, Lasota J, Foss R, Miettinen M (2009) Synovial sarcoma involving the head: analysis of 36 cases with predilection to the parotid and temporal regions. $\mathrm{Am} \mathrm{J}$ Surg Pathol 33: 1494-1503. [Crossref]

6. Farruggia P, D'Angelo P, Lo Cascio M, Solazzo L, Montemaggi P, et al. (2008) Synovial sarcoma of the neck in a child: a multidisciplinary approach. Pediatr Hematol Oncol 25: 431-437. [Crossref]

7. Thompson RC Jr, Garg A, Goswitz J, Cheng EY, Clohisy DR, et al. (2000) Synovial sarcoma. Large size predicts poor outcome. Clin Orthop Relat Res 18-24. Crossref]

8. Kartha SS, Bumpous JM (2002) Synovial cell sarcoma: diagnosis, treatment, and outcomes. Laryngoscope 112: 1979-1982. [Crossref]

9. Jay A, Hutchison I, Piper K, Farthing PM, Richards PS (2008) Synovial sarcoma presenting as a parotid mass: case report and review of literature. Head Neck 30: 16541659. [Crossref]

10. Ishiki H, Miyajima C, Nakao K, Asakage T, Sugasawa M, et al. (2009) Synovial sarcoma of the head and neck: rare case of cervical metastasis. Head Neck 31: 131135. [Crossref]

Copyright: (C2016 Wang CW. This is an open-access article distributed under the terms of the Creative Commons Attribution License, which permits unrestricted use, distribution, and reproduction in any medium, provided the original author and source are credited. 